\title{
A Velocity-Adaptive Handover Scheme for Mobile WiMAX
}

\author{
Caiyong HAO, Hongli LIU, Jie ZHAN \\ Department of Electronic Information Engineering, Hunan University, Changsha, China \\ Email: haocaiyong@gmail.com,hongliliu@vip.sina.com,jiezhanwl@163.com \\ Received March 7, 2009; revised July 27, 2009; accepted September 12, 2009
}

\begin{abstract}
Mobile WiMAX is a wireless networking system based on the IEEE 802.16e standard. In order to support mobile, some kinds of handover schemes must be adopted, and the hard handover is defined as mandatory. Since the data transmission should be paused during the hard handover process, it causes handover delay in mobile communication. The handover delay makes severe degradation in system performance when implemented in real-time applications such as IPTV and VoIP. The existing draft standard considers only the received signal strength when deciding handover initiation. However, the velocity factor also has an important influence on handover initiation and can not be neglected. To deal with these problems, this article proposes a velocity-adaptive handover scheme. This scheme adopts dynamic handover threshold according to different velocity to skip some unnecessary handover stages, reduces handover delay and enhances the network resource utilization. The simulation result and performance analysis validate the efficiency of the proposed scheme.
\end{abstract}

Keywords: Mobile WiMAX, Handover, Delay, Velocity-Adaptive

\section{Introduction}

In order to meet the demand of high data rate in wireless service for anytime, anywhere, and anyone, the Mobile WiMAX (Worldwide Interoperability for Microwave Access) based on the IEEE 802.16e [1] standard is developed for broadband wireless access as a promising technology. The IEEE $802.16 \mathrm{e}$ is the new, mobile version of the old WiMAX specification known as IEEE 802.16-2004 [2], which is a wireless, but fixed, data transmission scheme for providing broadband connection for metropolitan areas.

Support for handover (HO) is the most important amendment in IEEE 802.16e to embrace mobility. The $\mathrm{HO}$ is performed to maintain a continuous data transmission service for all applications when a Mobile Station (MS) is moving across cell borders of the BSs (Base Station). The IEEE802.16e defines three basic types of HO [3]: Hard handover (HHO), Macro Diversity Handover (MDHO) and Fast Base Station Switching (FBSS). MDHO and FBSS are soft handover. HHO is mandatory in WiMAX system while the others are optional ones. HHO adopt break-before-make scheme, the MS stops its radio link with the serving BS before establishing its radio link with the target $\mathrm{BS}$. This is a rather simple $\mathrm{HO}$ but causes long HO delay and service disruption for some applications, especially when the MS is in high velocity. Thus it is unsuitable for services requiring low latency. In MDHO or FBSS scheme, a MS is registered to several BSs at the same time. For MDHO, a MS communicates with two or more BSs in a given interval, while for FBSS, a MS communicates with only one BS. Since both the MDHO and FBSS adopt the make-before-break scheme, they can improve link quality and provide better performance for users. In MDHO and FBSS, the Diversity Set shall be maintained by the MS and the BS. Since the MS and the BS have to scan and modify the Diversity Set periodically, these schemes demand more capacity and multiple channels in terms of bandwidth efficiency, which give rise to wireless resource waste.

Up until now, a few papers have proposed several schemes to deal with the research about HO in IEEE 802.16e. In [4], fast handover scheme for real-time downlink services using fast DL_MAP_IE is suggested, which allow forward data transmission before the establishment of the MS registration and authorization. However, it is not adopted by IEEE $802.16 \mathrm{e}$ while some fast handover schemes such as soft handover are adopted. In [5], it proposed to associate only one neighboring BS instead of 
several BSs. But, [5] hasn't given an effective method to select only one neighboring BS. In [4] and [5], both neglected the HO threshold value's influence on $\mathrm{HO}$ process.

In this paper, a velocity-adaptive handover scheme is proposed to reduce the $\mathrm{HO}$ delay and the waste of the wireless network resource. In WiMAX system, the Received Signal Strength Indicator (RSSI) is typically used as a measure of signal quality. And as soon as the RSSI form the current serving BS is lower than a threshold and the RSSI from a potential target BS reaches a threshold, $\mathrm{HO}$ is executed. The most important factor to initiate $\mathrm{HO}$ is the received signal strength and MS mobility. However, the IEEE802.16e standard considers only the former. In our scheme, the HO threshold is set variably according to the MS's velocity, which can reduce the $\mathrm{HO}$ delay and wireless network resource waste.

The remainder of this paper is organized as follows. Section 2 describes the HO process in IEEE 802.16e. Then Section 3 proposes the velocity-adaptive handover scheme. And the simulation and the performance analysis of the proposed HO scheme are given in Section 4. Finally the conclusion is provided in Section 5.

\section{Handover Process and Analysis}

\subsection{Handover Process}

The HO process defined in the IEEE 802.16e consists of two phases. In the first phase, network topology acquisition is carried out before HO initiation. Then the actual $\mathrm{HO}$ process is performed, which includes HO decision, $\mathrm{HO}$ initiation, ranging and network re-entry stage. The detail explanations of the $\mathrm{HO}$ process are given as follows.

\subsubsection{Network Topology Acquisition}

The BS periodically broadcasts the network topology information using MOB NBR-ADV messages, which contains channel information of neighboring BSs such as the BS ID (Identifier), radiation power, and their UCD (Uplink Channel Descriptor) and DCD (Downlink Channel Descriptor) information. Thus, the MS is able to synchronize with neighboring BSs without listening their DCD / UCD broadcast messages. Once the MS synchronize with the neighbor BSs, it can start a scanning and association procedure in order to select a candidate BS for HO. The scanning procedure is done through exchanging MOB SCAN-REQ/RSP messages with the serving BS. During the scanning process, all downlink and uplink transmissions are paused and the MS can optionally perform association with the neighbor BSs by performing initial ranging. To acquire ranging parameter and service availability information for the purpose of selecting a potential future $\mathrm{HO}$ target $\mathrm{BS}$, the association
HO Process

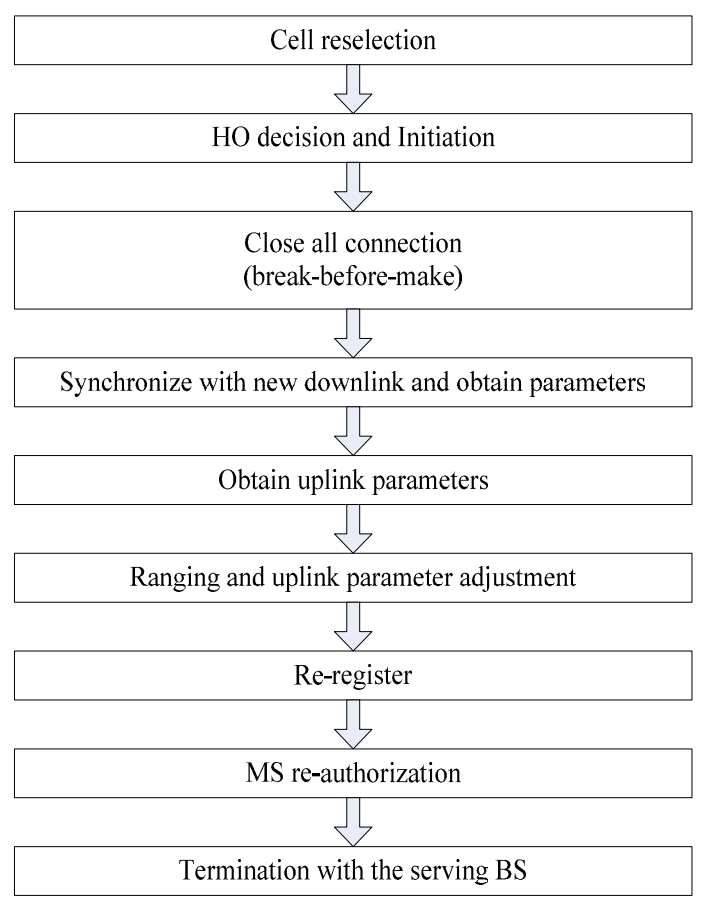

Figure 1. HO Process

procedure is performed between the MS and the candidate target. Initial ranging process performed during MS's scanning interval is optional. The MS can reuse this information for the future $\mathrm{HO}$ through the initial ranging values of associated $\mathrm{BS}$.

\subsubsection{Handover Process}

$\mathrm{HO}$ is executed when a MS migrates from the serving BS to the target BS. The HO process consists of the following stages and is illustrated in Figure 1.

\subsection{Handover Analysis}

At first, the MS conducts cell reselection with the information acquired from network topology acquisition to evaluate the potential target BSs. If the network topology has not changed during the process, this stage can be abbreviated. Such procedure does not terminate the existing connection to the Serving BS. Then HO Decision and Initiation is performed, which is the beginning of the actual HO. The HO Decision consummates with a notification of MS intent to handover through MOB_MSHOREQ or MOB_BSHO-REQ message. The HO is initialized, then the MS synchronize with target BS's downlink to obtain the downlink and uplink parameters. If the MS has received a neighbor advertisement earlier, the synchronization procedure can be faster. And if a HO notification was sent by the serving $\mathrm{BS}$ and received by the target BS via backbone connection, non-contention-based 
initial ranging can be assigned, which shortens the $\mathrm{HO}$ delay.

A good handover scheme should minimize the $\mathrm{HO}$ delay and reduce wireless channel resource waste. However, some factors degrading the system performance exist in the HO process. Suppose that the MS moves in a certain velocity, If the velocity is low, the network topology architecture may maintain the same in a long time, thus in the cell reselection stage, the MS can use the same network topology information and skip this stage. Also, since the received neighbor advertisement (include BS ID, DCD and UCD) do not change, the MS could synchronization to target BS downlink by performing non-contention-based initial ranging. Therefore, the HO delay is able to shorten. However, for a high velocity, the channel condition change frequently, which makes the pre-obtained information become useless. So, during the actual HO process, the neighboring BSs scanning and contention-based ranging operation must be performed, which causes a long HO delay and wireless channel resource waste. To deal with these problems the handover scheme should be adjusted with the velocity.

Once the alternate target BS has been successfully selected, before going into normal operation, network reentry process is initiated. It includes MS authorization and new BS registration. After the success registration with the target BS, the MS sends MOB_HO_IND message and notifies the serving $\mathrm{BS}$ that the $\mathrm{HO}$ is completed.

\section{Velocity-Adaptive Handover Scheme}

In this section, we propose the velocity-adaptive handover scheme. Firstly, we mainly focus on the HO decision. The Mobile WiMAX specification [6] defines the procedures during the $\mathrm{HO}$, but does not include the $\mathrm{HO}$ decision. Typically, the MS makes the HO decision according to signal quality, which can be measured by the RSSI. Figure 2 shows a simple case involving two BSs and an MS moving away from base station A (serving BS) toward base station B (target BS).

The $\mathrm{Th}_{\text {handover }}$ represents the $\mathrm{HO}$ threshold. And the $\mathrm{Th}_{\text {drop }}$ is the point below which the quality of the link becomes unacceptable, and will lead to excessive packet loss and the session being dropped. The hysteresis value $(\Delta \mathrm{H})$ is used to eliminate thrashing effect. The idea is that the MS choose those BSs with high RSSI value which results in a better link-level communication with the target BS and a lower bit error rate. Typically, HO procedures are initiated when the RSSI drops below the $\mathrm{Th}_{\text {handover }}(1)$. Also, HO is executed only if there is another BS for which the RSSI is at least $\Delta \mathrm{H}$ higher than the $\mathrm{Th}_{\text {drop }}(2)$. These can be described as fallows:

$$
R S S I_{\text {cur }}<T h_{\text {handover }}
$$

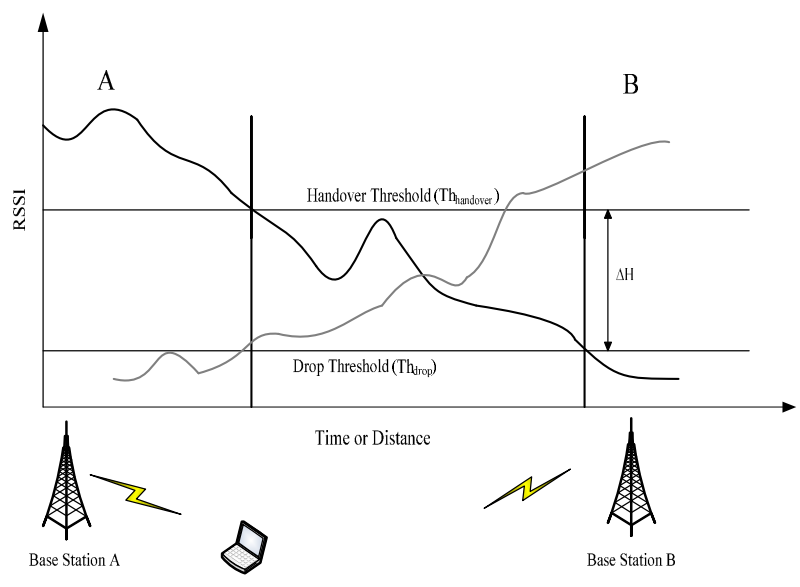

Figure 2. Handover decision based on RSSI.

$$
R S S I_{\text {candidate }}>T h_{\text {drop }}+\Delta H
$$

We have known that when the MS moves in a high velocity, the network channel information will change frequently, so that some unnecessary stages must be performed, which makes severe degradation in system performance such as $\mathrm{HO}$ delay and network resource waste. Form Equation (1) and (2), it can be known that if the $\mathrm{Th}_{\text {handover }}$ is set higher, the frequency of HO initiated will be higher, thus the network channel information can be acquired quickly. It means that the probability of pre-obtained information used in the HO process (cell reselection and synchronization to target BS downlink) will be improved. So it can reduce the HO delay. However, the frequently performing $\mathrm{HO}$ causes a great wireless network resource waste. If the $\mathrm{Th}_{\text {handover }}$ is set lower, the HO frequency becomes lower. Since the pre-obtained information do not update with the changes of channel condition, the neighboring BSs scanning and contention-based ranging operation must be performed. Therefore, the HO delay will be longer, but the wireless channel resource is consumed less. If the $\mathrm{Th}_{\text {handover }}$ maintains a constant value regardless of the velocity, for a high $\mathrm{Th}_{\text {handover, }}$ the wireless channel resource waste will increase in the low velocity condition, and for a low Thhandover, the HO delay will increase in the high velocity condition.

To keep the balance of the two facets and cope with HO delay and wireless channel resource waste problems, we propose the velocity-adaptive handover scheme. The scheme changes the HO threshold by dynamically adapting the $\mathrm{Th}_{\text {handover }}$ value based on velocity. It can be analyzed as follows:

$$
T h_{\text {handover }}=T h_{\text {drop }}+\Delta H
$$

According to the analysis above, $\Delta \mathrm{H}$ should become larger with the velocity increase. In the stationary state $(v=0)$, the network channel topology information keeps constant, and the HO delay is least, to avoid the unnec- 
essary network waste, the $\mathrm{Th}_{\text {handover }}$ should be setted as low as possible, so it might be the same value of the $\mathrm{Th}_{\mathrm{drop}}$, thus the $\Delta \mathrm{H}=0$ (when $\mathrm{v}=0)$. In the highest velocity situation ( $\mathrm{v}$ tends to infinity), the network channel topology information changes so quickly that the improvement of the $\mathrm{Th}_{\text {handover }}$ have nearly no effect on the HO delay, for some unnecessary stages must be performed. Thus the $\Delta \mathrm{H}$ will maintain a nearly constant value when in a very high velocity. In other situation, the $\Delta \mathrm{H}$ would be set larger with the velocity becomes higher. In order to reflect this dynamic character, an influence factor $r$ is proposed, which is the index associated with threshold value and velocity.

$$
\begin{aligned}
& \Delta H=r \cdot T h_{\text {drop }} \\
& r=\log _{2}(\mathrm{v}+1)
\end{aligned}
$$

where $\mathrm{v}$ is the velocity of the MS. It can be acquired from the received SINR [7]. To make the simulation simple, the $\mathrm{Th}_{\mathrm{drop}}$ is set as a constant value $2 \mathrm{~dB}$. Applying Equations (1), (2) and (3), the initial HO threshold $\mathrm{Th}_{\text {handover }}$ is given by

$$
T h_{\text {handover }}=T h_{\text {drop }}\left(1+\log _{2}(\mathrm{v}+1)\right.
$$

From the Equation (6), we can acquire that the $\mathrm{Th}_{\text {hand- }}$ over can be set different value according to the different velocity of the MS. When the MS moves in a higher velocity, the $\mathrm{Th}_{\text {handover }}$ is set higher, and thus the HO delay is reduced. When the MS moves in a low velocity, the $\mathrm{Th}_{\text {handover }}$ is set lower. Since the unnecessary HO is reduced, the wireless channel resource waste becomes little.

\section{Simulation and Performance Analysis}

In order to evaluate the performance of the proposed handover scheme, a simulation platform is built. The $\mathrm{HO}$ threshold has an influence on HO delay, which depends on the velocity of the MS. Table 1 shows the main parameters and the default values used in the simulation.

The simulation was done with MS speeds between 1 and $40 \mathrm{~m} / \mathrm{s}$ with $1 \mathrm{~m} / \mathrm{s}$ step. The $40 \mathrm{~m} / \mathrm{s}$ equals to $144 \mathrm{~km} / \mathrm{h}$, which is above the $100 \mathrm{~km} / \mathrm{h}$ limit described in IEEE 802.16e for a seamless handover. The Okumura-Hata model for small or medium city [8] was used for evaluating path losses.

When the MS is moving to the border of one BS in a certain speed, the signal quality of the Serving BS begins to degrade, if the signal level meets the Equation (5), initial HO process would be performed, and if the condition meets the Equation (6), the actual process of $\mathrm{HO}$ would be executed.

In the HO based on constant threshold scheme, we use the constant threshold value (Drop-threshold 2dB, Default handover-threshold $4 \mathrm{~dB}$ ) when the velocity varied.
Table 1. Simulation parameters.

\begin{tabular}{cc}
\hline Simulation Parameters & Value \\
\hline Handover type & HHO \\
Propagation model & Okumura-Hata \\
BS to BS distance & $800 \mathrm{~m}$ \\
Cell radius & $5000 \mathrm{~m}$ \\
Number of MS & 1 \\
Number of BS & 2 \\
Height of MS & $2 \mathrm{~m}$ \\
Height of BSs & $30 \mathrm{~m}$ \\
Frequency & $5 \mathrm{G}$ \\
Drop-threshold & $2 \mathrm{~dB}$ \\
Default handover-threshold & $4 \mathrm{~dB}$ \\
Step of simulation & $1 \mathrm{~m} / \mathrm{s}$ \\
Speed range & $1-40 \mathrm{~m} / \mathrm{s}$ \\
\hline
\end{tabular}

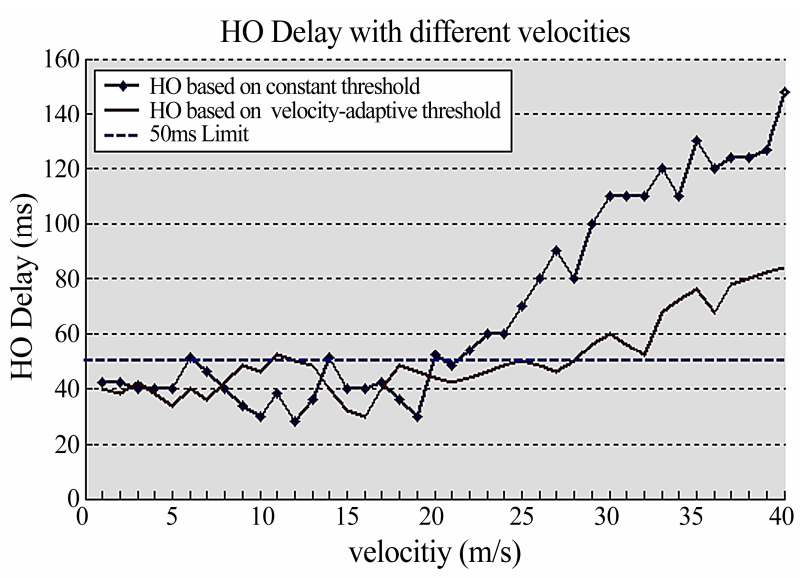

Figure 3. HO delay changes with the velocity.

And, we propose velocity-adaptive HO scheme which adopts different threshold value according to the different velocity (6).

The simulation results of constant threshold $\mathrm{HO}$ scheme and velocity-adaptive HO scheme are shown in Figure 3.

From Figure 3, we know that when the MS is in a low velocity (below the $20 \mathrm{~m} / \mathrm{s}$ ), there is little influence when implementing our scheme. However, when the MS moves in a high velocity (above the $20 \mathrm{~m} / \mathrm{s}$ ), the HO delay is obviously reduced in using velocity-adaptive scheme.

In $\mathrm{HO}$ based on constant threshold scheme, the $\mathrm{HO}$ delay remained below the 50ms limit (the WiMAX Forum defines that the Mobile WiMAX supports the HO delay should be less than $50 \mathrm{~ms}$ ) until the velocity rises up to $20 \mathrm{~m} / \mathrm{s}$, apart from a few exceptions that exceeded the limit only few milliseconds. As the velocity rises, the HO delay is growing up to $150 \mathrm{~ms}$.

In $\mathrm{HO}$ based on velocity-adaptive scheme, the $\mathrm{HO}$ delay is below the $50 \mathrm{~ms}$ limit until the velocity is up to $28 \mathrm{~m} / \mathrm{s}$. After that, the delay increase to $82 \mathrm{~ms}$ region with the $40 \mathrm{~m} / \mathrm{s}$ MS velocity. Since the high velocity causes a rapid changes of the network topology and wireless 
channel condition and the RSSI is not stable, some steps such as cell reselection and synchronize to downlink of the target BS may consume more time, so we can not always make the HO delay less than $50 \mathrm{~ms}$ in a high velocity, but in this condition our scheme reduces the $\mathrm{HO}$ delay greatly compared with the HO based on constant threshold.

\section{Conclusions}

In this paper, a velocity-adaptive handover scheme is presented. According to the existing draft version of 802.16e standard, the HO initiation should be performed if the RSSI of the serving BS is lower than the threshold. However, it does not consider the velocity's influence on the HO process, and the HO threshold is set as a constant. So the velocity has a bad effect on the HO performance. To cope with this problem, our scheme is proposed, the HO threshold is set variably according to the MS mobility. The simulation results show that HO delay below $50 \mathrm{~ms}$ limit when the velocity vary from $20 \mathrm{~m} / \mathrm{s}$ to $28 \mathrm{~m} / \mathrm{s}$. And HO delay is greatly reduced when the velocity exceeds $28 \mathrm{~m} / \mathrm{s}$. Therefore, this velocity-adaptive handover scheme can provide seamless communication for Mobile WiMAX in delay-sensitive and high velocity applications.

\section{References}

[1] IEEE P802.16e/D12, “Air interface for fixed and mobile broadband wireless access systems: amendment for physical and medium access control layers for combined fixed and mobile operation in licensed bands," 2005.

[2] IEEE Std 802.16-2004 (Revision of IEEE Std 802.162001), "IEEE standard for local and metropolitan area networks-Part16: Air interface for fixed and mobile broadband wireless access systems,” 2004.

[3] WiMAX Forum, Mobile WiMAX-Part I: "A technical overview and performance evaluation," 2006.

[4] S. Choit, G. H. Hwangt, et al., "Fast handover scheme for real-time downlink services in IEEE 802.16e BWA systems," IEEE Vehicular Technology Conference (VTC 2005), Stockholm, Sweden, Vol. 3, pp. 2028-2032, May 2005.

[5] D. H. Lee and K. Kyamakya, "Fast handover algorithm for IEEE 802.16e broadband wireless access system," IEEE Wireless Pervasive Computing Conference, pp. 16-18 January 2006.

[6] IEEE 802.16e-2005, IEEE Standard for local and metropolitan area networks-Part16: "Air interface for fixed and mobile broadband wireless access systems-amendent2: physical and medium access control layers for combined fixed and mobile operation in licensed band," 2006.

[7] M. D Austin and G. L Stuber, "Velocity adaptive handoff algorithms for microcellular systems," IEEE Transactions, Vol. 43, pp. 549-561, August 1994.

[8] T. Camp, J. Boleng, and V. Davies, "A survey of mobility models for ad hoc network research," Wireless Communicaitons and Mobile Computing (WCMC), Vol. 2, pp. 483-502, May 2002. 